\title{
More specific CRISPR editing
}

Studies from three independent groups address the problem of off-target genomic cleavage in the CRISPR-Cas9 system.

The ease with which a gene of interest can be modified using the clustered, regularly interspaced, short palindromic repeats (CRISPR) system has opened up the possibility of genome editing essentially at will to almost any research lab. All that is required is the expression, in the cell or organism of interest, of the Cas9 endonuclease and a guide RNA (gRNA) directing the nuclease to the target site.

But the specter of off-target effects that has plagued other targeted nucleases has raised its head for CRISPR as well. Several groups have reported that Cas9 can and does cleave at some off-target sites in the genome. Indeed, as recently reported by Mazhar Adli and colleagues at the University of Virginia, Charlottesville, Cas9 binds to many sites in the human genome other than the desired one.

Adli and colleagues conducted chromatin immunoprecipitation followed by sequencing (ChIP-seq) experiments in HEK293T cells to examine the binding of enzymatically inactive Cas9 (dCas9) genome wide for 12 different gRNAs. They found that the number of off-target binding sites depended on the gRNA. For most gRNAs, dCas9 bound at several (tens to hundreds) of off-target sites; dCas9 was also, as expected, highly enriched at the expected on-target site (Kuscu et al., 2014). Catalytically active Cas 9 cleaved some, although not all, of the off-target binding sites identified by ChIP-seq.

These genome-wide data confirm, at the level of Cas9 binding, that the CRISPR system may not have the specificity required for some applications. What is to be done? One strategy is to engineer Cas9 such that cleavage is dependent on dimerization, as for the zinc-finger nucleases (ZFNs) and transcription activator-like effector nucleases (TALENs). By requiring binding to two precisely disposed genomic half-sites, this approach should substantially reduce the number of sites that can serve as off-targets.

The groups of David Liu at Harvard University and Keith Joung at Massachusetts General Hospital now report the generation of such dimeric nucleases (Guilinger et al., 2014 and Tsai et al., 2014). To achieve this, the researchers fused dCas9 to the Fok 1 nuclease, the obligately dimeric nuclease used also in ZFNs and TALENs, both teams converging on fusion constructs in which the Fok1 nuclease is fused to the $\mathrm{N}$ terminus of inactive Cas9 (the opposite orientation to that in ZFN and TALENs).

In the optimal configuration, the on-target cleavage efficiency of these dimeric fusion constructs is only slightly lower than, or even comparable to, that of wild-type Cas9, both groups report. As expected, the requirement for two gRNAs, for precise orientation of the target sites, and for correct spacing between half-sites is more stringent for the Fok1-fusion architecture in comparison to the Cas9 nickase, a variant of Cas9 that cleaves only one DNA strand and that has also been proposed as an alternative strategy, in a paired configuration, to improve CRISPR specificity.

Using deep sequencing to examine previously identified off-target sites of wild-type Cas9, both the Liu and Joung groups report that the dimeric Fok1-dCas9 fusion has substantially less measurable off-target activity than the wild-type enzyme. Furthermore, Joung and colleagues observed measurable mutagenic activity of Cas9 nickases in the presence of even a single gRNA. Even though the more stringent sequence requirements of the dimeric enzyme will also reduce the number of available on-target sites, the researchers estimate that targetable sites should be present at a practically feasible frequency in the genome.

Dimeric Fok1-dCas9 fusions should prove a useful starting point towards genome editing that is not only crisp but also very specific.

\section{Natalie de Souza}

\section{RESEARCH PAPERS}

Guilinger J.P. et al. Fusion of catalytically inactive Cas 9 to Fok1 nuclease improves the specificity of genome modification. Nat. Biotechnol. 32, 577-582 (2014).

Kuscu, C. et al. Genome-wide analysis reveals characteristics of off-target sites bound by the Cas 9 endonuclease. Nat. Biotechnol. doi:10.1038/nbt.2916 (18 May 2014).

Tsai, S.Q. et al. Dimeric CRISPR RNA-guided Fok1 nucleases for highly specific genome editing. Nat. Biotechnol. 32, 569-576 (2014). 